\title{
Discrimination between internal faults and inrush current phenomena in power transformers
}

\author{
Bahram Noshad $^{1 *}$, Mohammad Sadegh Javadi ${ }^{2}$ and Ali Esmaeelnejad ${ }^{3}$ \\ $1^{*}$ Department of Electrical Engineering, Mahshahr Branch, Islamic Azad University, Mahshahr, Iran \\ ${ }^{2}$ Department of Electrical Engineering, Science and Research Branch, Islamic Azad University, Fars, Iran \\ ${ }^{3}$ Department of Electrical Engineering, Science and Research Branch, Islamic Azad University, Fars, Iran
}

BahramNoshad@yahoo.com,msjavadi@gmail.com, ali.esmaeelnezhad@gmail.com

\begin{abstract}
Conventional second harmonic current restriction approach is an adopted method which has been implemented to transformer differential protection schemes. Because of unreliable nature of this approach in discrimination between inrush current, internal faults and external faults and due to the saturation of current transformers, we present new features of the differential protection scheme. This helps to determine the feasibility and applicability of the proposed approach in differential protective scheme for power transformer. The Current Transformer Saturation (CTS) phenomenon issue and its revelation is one of the most significant problems in power system protection particularly in the differential protection. In this paper, the saturation phenomenon and its effects on the differential protection are analyzed. Prevalent algorithms in digital signal processing which are implemented in power system cannot correctly recognize this phenomenon. Mal-operation of differential protection relay takes place when saturation in CT is appeared. This paper presents a Third-Difference Algorithm (TDA) for CTS recognition. In order to make comparison between the presented approach and prevalent digital protective algorithm, an identical case study is simulated. Simulation results also show that the TDA is both satisfactory and consistent with expectation.
\end{abstract}

Keywords: Current transformer, Saturation phenomenon, Third-difference Algorithm, Internal and external faults.

Introduction

Current transformer is a prevalent phenomenon caused by asymmetry of the conductance geometric arrangement which occurred in core design. This phenomenon particularly in earth fault current transformers has much more importance because of their high sensitivity.

During a fault, the secondary current signals may be distorted by CST which could result in the mal-operation of a protection relay. Thus, protection relays are designed to cope with the effect of partial saturation, but the operating performance is a compromise between dependability and security, and an appropriately designed CT must be used (Kang et al., 2003).

When the core of a CT enters saturation, the magnetizing inductance reduces to a small value, the excitation current becomes significant and the wave shape of the secondary current is distorted. Points of inflection occur in the secondary current wave shape when the CT enters and leaves saturation.

Bunyagu et al. (2001) proposed a CT saturation detection method based on the evaluation of the mean of the error and the mean and variance of the current amplitude (Bunyagul et al., 2001). The error is derived assuming the current is a perfect sinusoid and consequently the summation of the current and its second order derivative is zero.

A method for detecting the onset of CT saturation was suggested by Phadke \& Thorp (1988). It relies on the collapse in current that occurs when the CT enters saturation. It can detect only the onset of saturation if the magnitude of the first-difference function exceeds the threshold. Difficulties may arise if the current does not small value at the start of saturation.

An algorithm for calculating the core flux using the secondary current was suggested by Kang et al. (1997). The flux is used to calculate the excitation current, which is then used to compensate the distorted current. Simulated results were correct, but relied on the assumption that the residual flux was zero when the fault occurred (Kang et al., 2004).

In this paper, the CT transient model caused by fault current in primary windings is addressed. In this model the inducted voltage in secondary is calculated using basic electromagnetic analytical equations in transient and it is analyzed in discrete space using digital signal processing algorithms. Then the CTS phenomenon and its impact on the power system protection analyzed. Analytical equations corresponding to CTS are also presented. Prevalent digital signal processing algorithms are assessed and their advantages and disadvantages are introduced. Mal-operation in differential protection belong with this problem is also studied in this study.

\section{CT saturation phenomenon}

The relay necessary signals to operate are generally voltage and current which are transmitted from the power system to the protection system by voltage and current transformers, respectively. In this situation, operating point of a CT becomes more complicated than VT because of the aforementioned phenomenon. The accurate CTS algorithm for recognizing intensive short circuit currents and its effect on power system protection is important issue in power system relaying (Fernández, 2001). Saturation in CTs has a transient nature which is 
Fig. 1. Magnetizing characteristics of transformer core (Kitayama \& Nakabayashi, 2002)

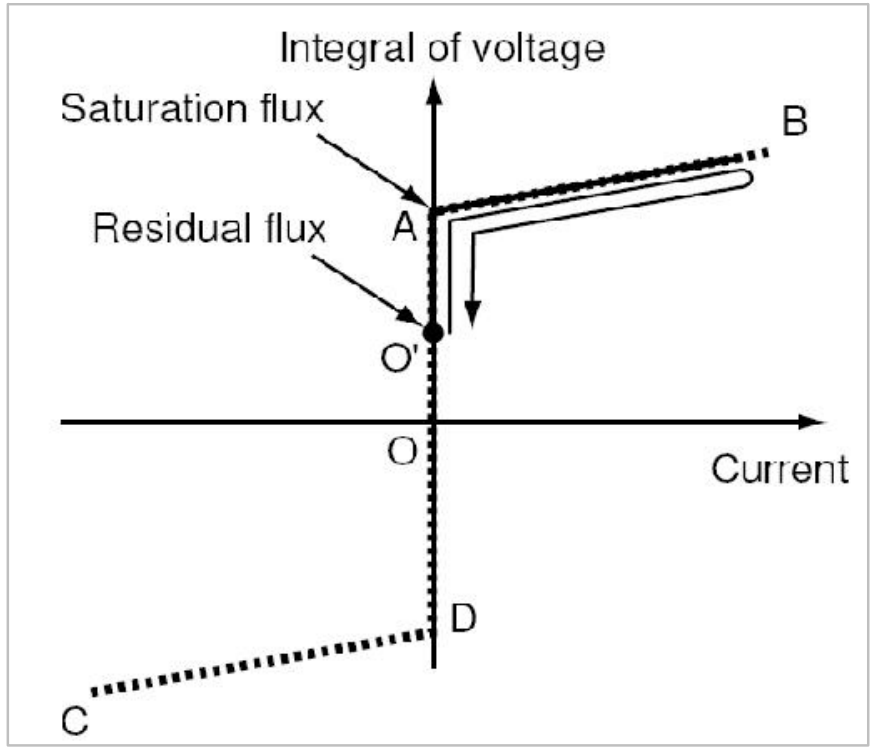

generally occurred in giant motors start up or when an intensive short circuit occurs.

The magnetizing characteristic of a transformer core are indicated by $\mathrm{B}-\mathrm{H}$ curve as shown in Fig. 1. The magnetic field intensity $H$ is in proportion to the current value $i$, and the change in magnetic flux density $B_{r}$ is in proportion to the integral of voltage $v$. the $\mathrm{B}-\mathrm{H}$ curve inherent to each transformer can be divided into two linear segments, one is segment $A-D$, the condition in which the transformer core is not saturated and the others are segments $A-B$ and $C-D$, the condition in which the transformer core is saturated because the magnetizing current in the non-saturated condition is negligible small (Kitayama \& Nakabayashi, 2002).

CTs used for protection have a different saturation curve comparing to ones used for measurement. Fig. 2

Fig. 2. Saturation curves of protection and measurement CTs.

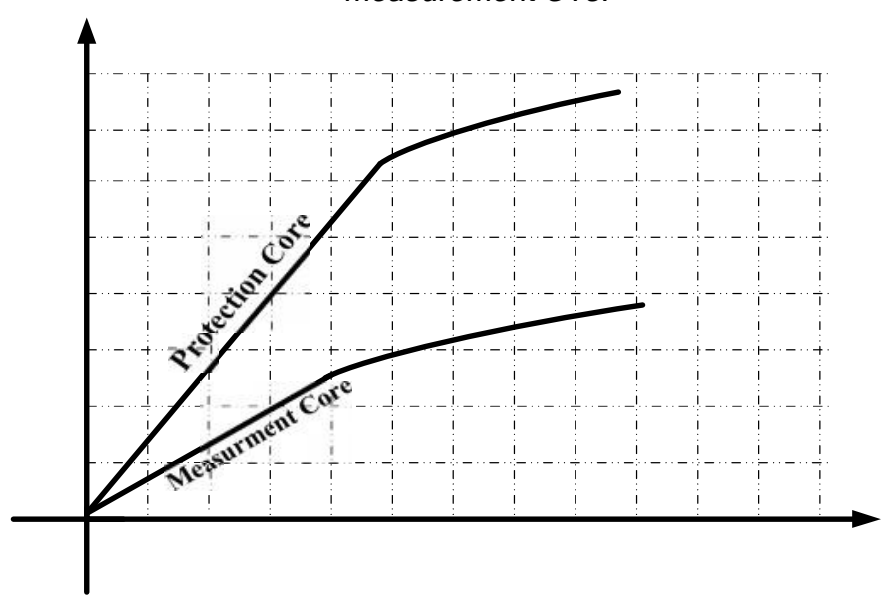

Fig. 3. Differential protection and the saturation of one of CTs in the short beyond the protective zone

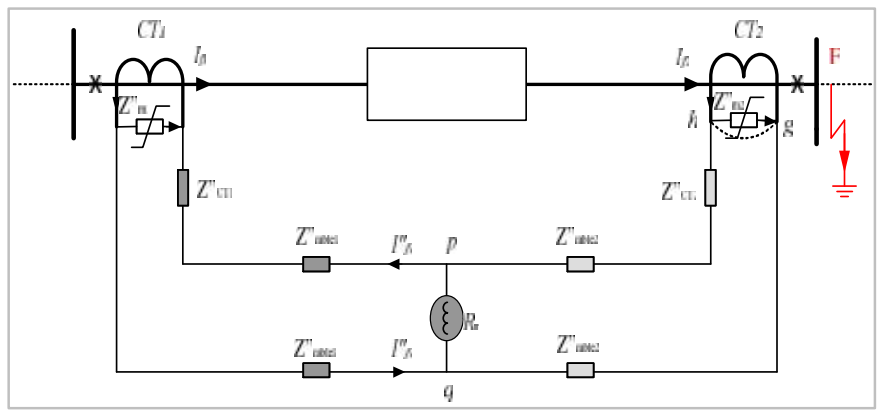

Fig. 4. Equivalent circuit for high impedance relaying within the fault beyond of the protective zone

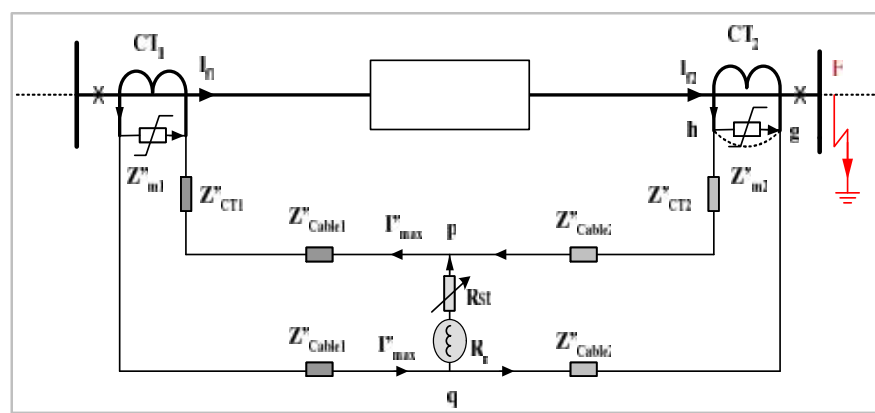

shows the saturation characteristics of these two categories of CTs.

Current transformer saturation has the most effect on the power system relaying, specifically in differential protection. The most significant factor in differential protection is considering the current of relay terminals and the protection system operation against it.

To prevent the protection system mal-operation current in saturation in traditional differential protection system, the high impedance differential protection scheme is used (Phadke \& Thorp, 1988).

\section{High impedance differential protection scheme}

The generic scheme of the differential protection without preventive circuit is shown in Fig. 3. For short circuit out of protective zone, if one of CTs became saturated for any reason, the secondary current output of CT would be zero; it means that the CT is shorted. In fact, for CT in full saturation condition, the secondary current would be zero and the total primary current passes through the magnetizing branch. In other words, the magnetizing branch inductance is very small and the magnetizing branch absorbs almost the complete secondary current and no current will meet the relay. The $\mathrm{g}$-h impedance of equivalent circuit considered zero in saturation.
Research article

COIndian Society for Education and Environment (iSee)
B.Noshad et al. Indian J.Sci.Technol. 
Fig. 5. Transformer equivalent circuit for power system transient analysis

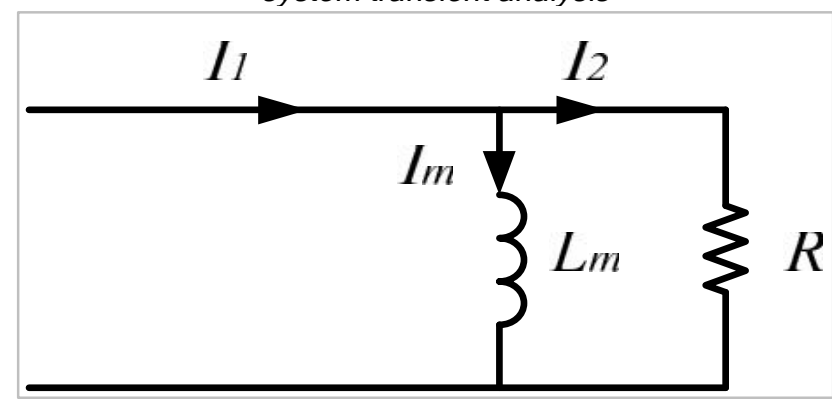

Fig. 6. Current signal wave of the CT secondary in normal condition ( $X$ axis is represented as number of samples)

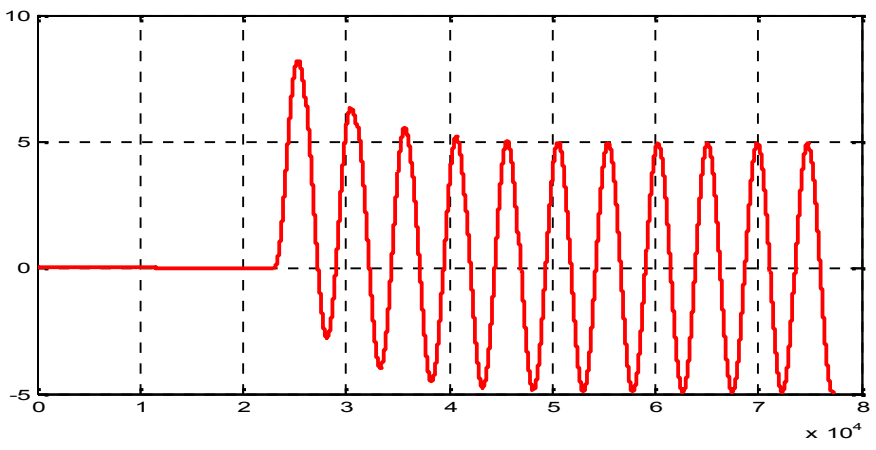

Fig. 7. Saturated current signal wave of the CT secondarv

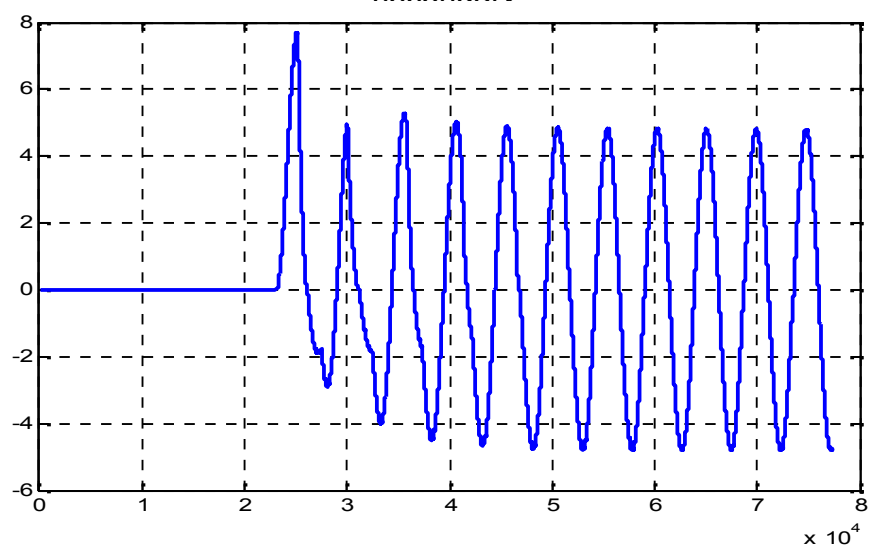

In Fig.3, it is supposed that the secondary CT (CT2) is saturated for short circuits beyond the protective area while primary CT (CT1) operates in normal condition. The CT 2 saturation means that nodes $h$ and $g$ are shorted. In this situation, CT1's output current has two paths in node $g$ to pass through. One through the relay circuit and the other one closes the saturated $\mathrm{CT}$, which its magnetizing impedance is zero. If the low impedance relay is used, the passing current through the relay would be so great that is more than the relay necessary current to operate and leads to relay mal-operation. To prevent the relay mal-operation under these conditions, one suggestion is offered which is impedance increase of relay path. The added impedance is something that the relay passing
Vol. 5 No. 7 (July 2012)

ISSN: 0974- 6846

Fig. 8.Prodar 70 algorithm response encountering

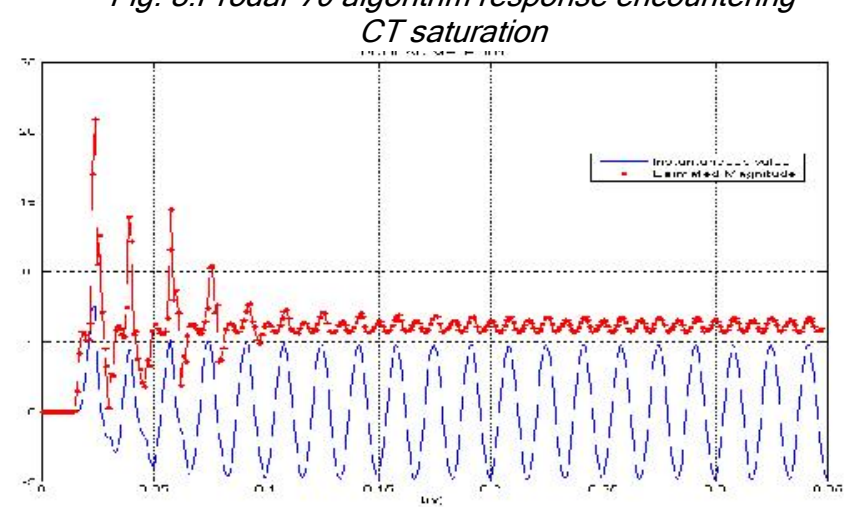

Fig. 9. DFT algorithm response encountering CT saturation

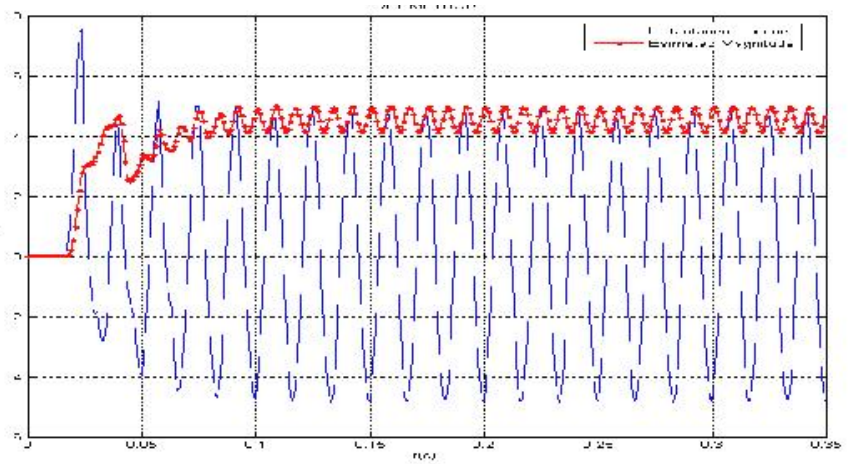

current in the worst conditions will be less than the relay necessary current to operate. To increase the impedance through the relay path, an adjustable resistor is used. Because adding the resistor stabilizes the differential protection for out of area short circuits, this resistor called stabilizer resistor (Rst).

Another method is using voltage relays having high impedance. The differential protection scheme, which the relay circuit impedance is high, has been known as high impedance scheme. This scheme is commonly used in Europe for bus protection or power transformers, generators, reactors and earth fault protection. In this section, the generic description of this scheme will be explained ignoring its applications.

High impedance protection system is a simple technique which requires that all CTs, used in the protection scheme, have relatively high knee point voltage, similar magnetizing characteristic and the same ratio. These CT shall be installed in all ends of the protected object. In order to make a scheme all CTs belonging to one phase shall be connected in parallel. From the CT junction points a measuring branch is connected. The measuring branch is a series connection of one variable setting resistor $\left(R_{S}\right)$ with high Ohmic value and over-current relay. Thus, the high impedance differential protection responds to a current flowing through the measuring brunch.

However, this current is result of a differential voltage caused by this parallel CT connection across the
Research article

CCIndian Society for Education and Environment (iSee) 
measuring branch. This current and voltage are interrelated by Ohms Low (A.B.B Doc., 2007).

High Impedance Protection for Short Circuits out of Area

Fig. 4 shows a high impedance protective scheme including a stabilizer resistor $\left(R_{\mathrm{st}}\right)$ series with low impedance over-current relay. In high impedance protection, the cables impedance connecting current transformer to the relay is shown as a resistor series device. Connecting cables impedance of both sides in each path are respectively shown with $Z_{\text {cable }}$ which is considered Ohmic in calculation for more simplicity. The CT's secondary equivalent impedance modeled as a resistor too.

Other parameters in Fig. 4 are as follows:

$I_{e}$ : CT's magnetizing current which is a small value if the $C T$ is not saturated.

\section{$Z_{m}^{\prime}$ : CT's magnetizing impedance}

The magnetizing impedance and its corresponding current depend on CT's core magnetic design and arrangement and the magnetizing curve normally used to calculate the magnetizing current. The maximum out of area current is calculated in order to get the circuit in the worst condition. When CT2 is saturated in the short circuit, the secondary output current equals to zero. In the saturated CT equivalent circuit, point's $g$ and $h$ is short circuit as mentioned above. The CT1 secondary current divided between the relay path and the CT2 secondary. The current passing through the relay under this condition is as follows:

$$
I_{r}=I_{\max }^{\prime \prime} \frac{\left(2 Z_{\text {cable } 2}+Z_{C T 2}^{\prime \prime}\right)}{\left(2 Z_{\text {cable } 2}+Z_{C T 2}^{\prime \prime}+R_{s t}+R_{r}\right)}
$$

The condition of the relay not operation is that the passing current is less than the adjusted value:

$$
I_{r}<I_{\text {set }}
$$

The stabilizer resistor can be calculated using (1) and (2). It is obvious that similar calculations must be presented for CT1 saturation condition that eventually the stabilizer resistor is calculated.

\section{High Impedance Protection for Internal Faults}

Although the impedance increase in the relay circuit stabilizes the protection scheme against out of area short circuit, the high impedance existence in CT's secondary leads to CT saturation because of overloading. The saturated secondary of CT, voltage under this condition would not be much more than the saturated voltage $V_{k}$ (saturation curve knee point voltage). It is obvious that to operate the high impedance protection, the voltage should be more than stabilization voltage of relay terminals $(p-q)$. To ensure that the necessary voltage of CT's terminals to operate the high impedance relay is created, it is necessary that the CT's saturation beginning start voltage be more than the stabilization voltage with the minimum margin pointed in (2) $t=n T$.

\section{Vol. 5 No. 7 (July 2012) ISSN: 0974-6846}

The results indicate that the proposed sensitive differential protection relay will remain stable during a high current external fault.

$V_{K}>2 \times V_{\text {set }}, V_{s e t}>V_{p q-\max }$

It is necessary that following inequalities should be satisfied for high impedance voltage relay.

The more the knee point voltage rather than the necessary voltage to operate the relay, the faster and more reliable the relay operation. Notice that from the short circuit occurrence in the area to the time that it is completely cleaned by the bus protection relay operation and circuit breakers opening, the CT must be saturated because of high impedance. If CTs were saturated, its operation would not be linear anymore and the voltage wave would be as positive and negative half cycles with impulse is would be appeared.

\section{Current Transformer Analysis in Transient}

Fig. 5 shows a transformer equivalent circuit in power system transient analysis. In the equivalent circuit, currents $i_{1}$ and $i_{2}$ can be considered as following. It also should be noticed that the $I_{1}, I_{2}$ and $I_{m}$ represented in frequency domain (Phasor). In time domain, primary current is:

$$
i_{1}(t)=I_{\max }\left[\operatorname{Cos}(\omega t-\theta)-e^{-\frac{t}{T_{p}}} \operatorname{Cos} \theta\right]
$$

And for secondary current we have:

$i_{2}(t)=A e^{-\frac{t}{T_{s}}}+B e^{-\frac{t}{T_{p}}}-C \sin (\omega t-\theta-\phi)$

In discrete time domain by sampling rate $T$, we can state

In above equations, $T$ equals to the sampling rate in each cycle. The secondary current is as shown in Fig. 6 if the CT is not saturated: But if the CT is saturated, the secondary current is as shown in Fig.7.

\section{Digital Signal Processing Algorithms in Saturation} Recognition

There are several digital processing algorithms presented to estimate the power system signals which separated into long-window and short-window algorithms. Some algorithms such as Prodar 70, Man-Morison are short-window algorithms and algorithms such as full wave and half wave Fourier algorithms, least square algorithm, etc. are long-window ones .

Although short-window algorithms have a fast response, their transient responses are not appropriate; while long-window algorithms' problem is their inertia.

Prodar 70 simulation as a short-window and DFT simulation within an identical saturated signal are presented in Fig. 8 and 9 respectively.

Third-difference Algorithm and its concepts for differential protection relaying are analyzed in next section. The aforementioned algorithm is a fast one with a desirable transient response which has simultaneous short-window and long-window algorithms features, Indian J.Sci.Technol. 
means high speed in recognition and transient response.

Conclusion

In this paper, the differential protection operation and traditional protection systems' problems in CT local saturation and its mal-operation for out of area fault or not operation for the fault in the region of protection are completely described because the differential protection is a unit protection, its correct protection for out of area is crucial. The CT saturation issue is a significant issue in differential protection system mal-operation. The commonly used digital algorithms in revealing the CT saturation are quite incapable. So, triple-differential algorithm to reveal the CT saturation presented as a discriminative approach for recognition the internal faults and inrush current phenomena in power transformers and its operation contributes to improve the accordant algorithm.

\section{Acknowledgements}

This work was extract of research entitled by "Discrimination between Internal Faults and Inrush Current Phenomena in Power Transformers" which is granted and supported by the Mahshahr Branch, Islamic Azad University, Mahshahr, Iran.

\section{References}

1. BunyagulT, Crossley P and Gale P (2001) Overcurrent protection using signals derived from saturated measurement CTs.IEEE PES 2001 SM, Vancouver, BC, Canada.

2. Document ABB (2007) Applying high impedance differential protection with IED 670. Application Example. 1-12, $A B B$.

3. Fernández C (2001) An impedance-based CT saturation detection algorithm for bus-bar differential protection. IEEE Trans. Power Delivery. 16(4), 12031209.

4. Kang YC, Kang SH and Crossley $P$ (2003) An algorithm for detecting CT saturation using the secondary current third-difference function. IEEE Bologna Power Tech Conf., Italy.

5. Kang YC, Wo. L, Jhong. E. (2004) Design and evaluation of an algorithm for detecting current transformer saturation. IEE Proc. Gener. Transm. Distrib., 151 (1), 27-35.

6. Kang YC, Wo. L, Jhong. E. (1997) An algorithm for compensating the secondary current of current transformers. IEEE Trans. Power Delivery. 12 (1), 11624.

7. Kitayama M and Nakabayashi M (2002) A new approaach to fast inrush currenrt discrimination based on transformer magnetizing charachtristic. 14th PSCC (Sevilla). pp: 1-7.

8. Phadke AG and Thorp JS (1988) Computer relaying for power systems. Research Studies Press LTD. 